\title{
Cognitively and socially induced stress affects postural control
}

\author{
Michail Doumas $^{1}\left[\right.$ Kinga Morsanyi ${ }^{1} \cdot$ William R. Young $^{2}$
}

Received: 15 August 2017 / Accepted: 8 November 2017 / Published online: 14 November 2017

(c) The Author(s) 2017. This article is an open access publication

\begin{abstract}
Postural control is an adaptive process that can be affected by many aspects of human behavior, including emotional contexts. The main emotional contexts that affect postural control are postural threat and passive viewing of aversive or threatening images, both of which produce a reduction in postural sway. The aim of the present study was to assess whether similar stress-related changes in postural sway can be observed using stress induced by social evaluative threat (SET) while performing arithmetic tasks. Twelve young adults performed an arithmetic and a postural control task separately, concurrently, and concurrently with added time pressure in the arithmetic task. In the final condition, participants were given negative feedback about their performance in the arithmetic task and performed it again while being observed (SET condition). Results showed that stress increased linearly with task demand. Postural sway and reaction times were not affected by the first two conditions; however, when time pressure was introduced, reaction times became faster and sway amplitude increased. Finally, introduction of SET caused the predicted reduction in postural sway and an increase in reaction times relative to the time pressure condition. Our results suggest that stress induced using a combination of arithmetic tasks and social evaluative threat leads to systematic changes in postural control. The paradigm developed in the present study would be very useful in assessing interactions between cognition, stress, and postural control in the context of postural instability and falls in older adults.
\end{abstract}

Keywords Postural control · Social Evaluative Threat $\cdot$ Mathematical Anxiety $\cdot$ Stress $\cdot$ Arithmetic $\cdot$ Processing Efficiency Theory

\section{Introduction}

Maintaining upright stance, or postural control, is an adaptive process that relies on sensory, motor and cognitive processes (Balasubramaniam and Wing 2002) and can also be affected by emotional contexts (for a review, see Hagenaars et al. 2014). An emotional context that has been extensively studied in postural control is postural threat, which is a situation imposing increased challenge on balance. Postural threat has been primarily assessed in a series of studies by Carpenter and colleagues (Carpenter et al. 1999, 2001; Adkin et al. 2000) who showed that when participants stood on the edge of an elevated surface (0.8-3.2 m high), real, or virtual (Cleworth et al. 2012); they exhibited a reduction

Michail Doumas

m.doumas@qub.ac.uk

1 School of Psychology, Queen's University Belfast, 18-30 Malone Road, Belfast BT9 5BN, UK

2 Department of Clinical Sciences, Brunel University London, Uxbridge, UK in postural sway and posterior body movement away from the platform's edge compared with standing at ground level. This sway reduction was accompanied by an increase in sway frequency, muscle co-contraction, and ankle stiffness. Standing on an elevated surface also induced increases in fear of falling, sympathetic arousal, and stress response (Cleworth et al. 2012; Horslen et al. 2014). More recent studies focused on the underlying mechanisms of this adaptive response and showed that postural threat is likely to induce changes in two of the sensory channels involved in postural control, proprioceptive (Davis et al. 2011; Horslen et al. 2013), and vestibular (Horslen et al. 2014; Lim et al. 2017).

A reduction in body movement as a response to emotionally engaging, fear-inducing stimulation, however, is not unique to postural-threat contexts. Fear responses and their underlying mechanisms have been studied in animals and have been classified into two categories: defensive action, characterised by fight or flight behavior in response to impending attack, and defensive immobility, characterised by freezing, bradycardia, and hyper-attentiveness (Lang et al. 2000). For example, fear-freezing, measured as the 
amount of time rats in a cage were not moving has been observed in the context of Pavlovian-conditioned aversive responses using an auditory stimulus (LeDoux et al. 1988).

Using fear-freezing responses in animals as a starting point, freezing has also been assessed in humans using a paradigm comprising passive viewing of aversive or threatening images, for example, images of mutilation (Azevedo et al. 2005; Facchinetti et al. 2006). Similar to posturalthreat research, these studies showed a reduction in sway, an increase in mean power frequency of sway but also bradycardia, the latter also being associated with freezing responses in animals. Similarly, Roelofs et al. (2010) showed a freezing response in reaction to angry faces, reflected in reduced heart rate and body sway, and Hillman et al. (2004) showed a backward body movement away from unpleasant stimuli in women which was not observed in the case of pleasant or neutral stimuli. This response was also affected by the previous experience of an aversive life event, with individuals who had experienced such an event showing a greater reduction in sway when exposed to aversive images compared with a control group (Hagenaars et al. 2012). However, another study manipulating arousal and valence using this paradigm (Horslen and Carpenter 2011) showed that only arousal affected postural sway similar to postural threat, and identified methodological limitations in some of the passive-viewing studies. The primary limitation involved the short duration of the postural trials $(<10 \mathrm{~s})$ in some of these studies (Hillman et al. 2004; Stins and Beek 2007; Roelofs et al. 2010), which was not long enough for the full range of time scales present in postural sway time series to be identified (Van der Kooij et al. 2011).

Despite the methodological limitations identified in studies of freezing in humans, the consensus in the literature is that fear-related postural responses can be induced by at least two different types of emotion-specific paradigms, postural threat, and passive viewing of aversive or threatening images. Given that these two paradigms induce similar sway reduction responses, it would be reasonable to assume that this reduction is a general effect that goes beyond the two paradigms and could also be caused by other emotion-specific manipulations. In the psychology literature, fear-related emotional responses are primarily triggered using socially induced stress and anxiety (Dickerson and Kemeny 2004).

A well-established, effective method of inducing high levels of stress in humans is the presence of social evaluative threat (SET), in tasks primarily including mental arithmetic, public speaking, and singing (Kirschbaum et al. 1993; Frisch et al. 2015). SET is characterised by emotional responses observed during tasks performed in circumstances, where an evaluative audience or a negative social comparison is present. For example, SET has been successfully used to induce stress in combination with a mental arithmetic task, the Montreal imaging stress task (Dedovic et al. 2005). This study asked participants to perform arithmetic calculations and used a mock performance indicator combined with negative feedback by both the task software (after each trial) and the experimenter (between blocks, which was the SET element). When negative feedback was provided, an increase in cortisol was observed relative to the control and rest conditions, suggesting an increase in stress. Furthermore, a metaanalysis of over 200 studies showed that SET, together with uncontrollability, induced the largest increases in cortisol levels and the longest times to recovery compared to all other stressors (Dickerson and Kemeny 2004), thus making SET a very effective way of inducing stress in humans. The high effectiveness of this method makes it an excellent candidate to use as a novel manipulation to induce stress in the context of postural control, to see whether posturalthreat- and passive-viewing-induced sway reduction can also be observed using SET-induced stress. Furthermore, performance of a mental arithmetic task while standing has been shown to affect both postural control and physiological arousal as measured by skin conductance (Maki and McIlroy 1996). Together, evidence suggests that SET in combination with mental arithmetic causes an increase in stress (Dedovic et al. 2013) and that mental arithmetic tasks affect physiological arousal and postural control (Maki and McIlroy 1996). However, a combination of these three tasks has not been previously used to induce stress in a postural control context.

The aim of this study was to assess whether a reduction in sway is observed when stress increases using a combination of mental arithmetic and SET manipulations. To this end, first, we assessed postural control and arithmetic separately and then concurrently. Subsequently, to increase stress and task demands, we added an element of time pressure by introducing a progress bar based on participants' own performance in the arithmetic task. It was expected that under time pressure, participants would allocate more resources to the arithmetic task in an attempt to perform it more efficiently. Finally, to increase stress and task demand further, we added an SET manipulation, providing negative performance feedback. This approach was used to incrementally increase task demands and stress in each condition. This incremental increase was used to ensure that we could assess the contribution of each component of our design (adding the arithmetic task, time pressure, and SET) on stress, postural control, and performance on the arithmetic task.

Theories of anxiety and cognition, such as the processing efficiency theory (Eysenck and Calvo 1992) and its successor, attentional control theory (Eysenck et al. 2007), predict that during relatively simple tasks, performers can compensate for anxiety-related inefficiencies in processing information through increasing cognitive effort. However, as task demands increase, such inefficiencies can no longer be compensated for and performance starts to show deficits. We 
predicted that self-rated stress would increase incrementally with task demand. More importantly, we predicted that this increase in stress following the SET manipulation would be accompanied by a reduction in postural sway reflecting a stiffening or freezing response in line with the previous research, (Adkin et al. 2000; Azevedo et al. 2005; Facchinetti et al. 2006; Roelofs et al. 2010). These findings would suggest that the reduction in postural sway observed when using postural threat and aversive images can also be induced using cognitively and socially induced stress.

Performance on the arithmetic task was expected to improve following the inclusion of a time pressure manipulation, due to recruitment of additional resources to, or prioritization of the arithmetic task. However, we predicted that performance in this task would eventually decline under high levels of stress and task demands (e.g., Brooks 2014) as a consequence of anxiety-related reductions in processing efficiency. More specifically, we predicted that only in trials containing increased task demands and associated higher stress (i.e., trials including SET), would individuals demonstrate significant reductions in postural sway in conjunction with reduced performance in the cognitive task.

\section{Method}

\section{Participants}

Twelve adults, eight females, and four males (mean age 20.7, SD 1.9 years) volunteered to participate in this study. Participants were undergraduate psychology students and received course credit for their participation. They reported no major neurological or musculoskeletal disorders and no intake of medication that affects postural control, for example, sleep medication or antidepressants (de Groot et al. 2013). All participants provided written informed consent and the study was approved by the School of Psychology, Queen's University Belfast ethics committee.

\section{Apparatus and tasks}

The study comprised a postural control task and an arithmetic task performed separately and concurrently. In the postural control task, participants were asked to maintain upright standing, barefoot with eyes open on the dual force plates of a Neurocom Smart Balance Master system (Natus Medical inc.) in 2-min blocks. Stance width was determined by the system's manufacturer and was $26 \mathrm{~cm}$ for height $<165$ and $30 \mathrm{~cm}$ for height $>165 \mathrm{~cm}$. During the task, participants were asked to wear a safety harness which ensured safety in case of loss of stability. No losses of stability were observed in this study. In all posture blocks, the standing surface was tilted in the anterior-posterior (toes down, toes up) direction in proportion to body sway, using support-surface sway reference (Black et al. 1983; McCollum et al. 1996). Sway reference is a well-established method of increasing postural sway by means of reducing proprioceptive information about body sway (Peterka and Black 1990; Peterka and Loughlin 2004; Doumas and Krampe 2010). For example, when participants sway 1 degree forward, the surface was tilted 1 degree down thereby maintaining a relatively constant ankle angle and inducing inaccurate proprioceptive information about body sway. In the present study, we used sway referencing in the AP direction with a gain factor of 2 , in which body sway of $1^{\circ}$ induces surface tilt of $2^{\circ}$. We used a higher gain to induce larger AP sway magnitude, because in young adults with eyes, open sway referencing at gain 1 is not as effective in inducing an increase in postural sway compared with eyes closed (Peterka and Black 1990; Clark and Riley 2007). We opted for using this method instead of quiet standing on a fixed surface, because we aimed to induce a certain amount of sway first, to increase the possibility of detecting a reduction in sway when SET was introduced. Vertical forces applied by the body on the force plates were recorded at a sampling frequency of $100 \mathrm{~Hz}$ and were used to derive the center of pressure (COP) time series in the anterior-posterior (AP) direction.

Participants performed an arithmetic verification task (Fig. 1a) both seated and standing. When seated, stimuli were displayed on a 19" computer screen (resolution: $1024 \times 768$ pixels), located $60 \mathrm{~cm}$ in front of the participant and $100 \mathrm{~cm}$ from the ground and when standing, they were displayed on a 17" monitor positioned at the participants' eye level, with the same resolution, embedded in the Neurocom system's surround (Fig. 1b). The task was presented with and without added time pressure. In the case of presentation without time pressure, stimuli were presented as white numbers (font size 48) on a black background and comprised a simple addition of a single-digit to two-digit number, or of two three digit numbers, followed by the sum (e.g., $14+3=17$ or $342+539=881$ ). Two types of problems were used to make the task less repetitive. A new set of problems was used in each block. Participants were asked to respond by pressing the left button of a wireless mouse if the sum was correct and the right if it was incorrect, as quickly and as accurately as possible. Arithmetic task stimuli were presented in blocks comprising 32 trials each, equally divided into correct and incorrect sums, and into smaller $(1+2$ digits $)$ and larger $(3+3$ digits $)$ pairs of numbers. If no response was provided after $10 \mathrm{~s}$, the trial was interrupted and the next trial was presented.

In the case of presentation with time pressure, stimulus presentation was the same, but a progress bar was introduced at the bottom of the screen (Fig. 1a), starting with a green part, followed by a dividing white line and a longer red part, with a constant total duration of $10 \mathrm{~s}$. The bar was 
Fig. 1 Experimental setup. a Arithmetic task display, b performing the task while standing and $\mathbf{c}$ experiment timeline. SET social evaluative threat
A

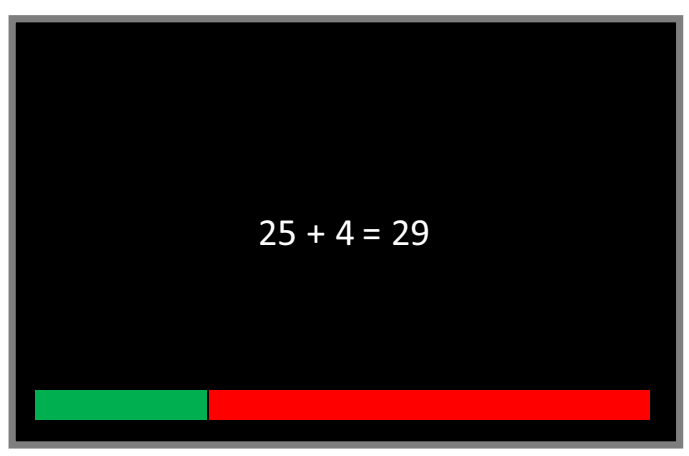

B

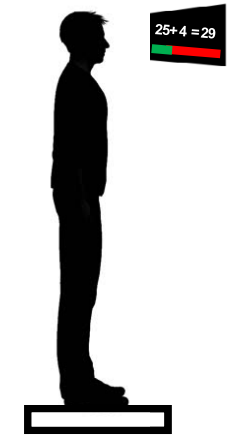

C

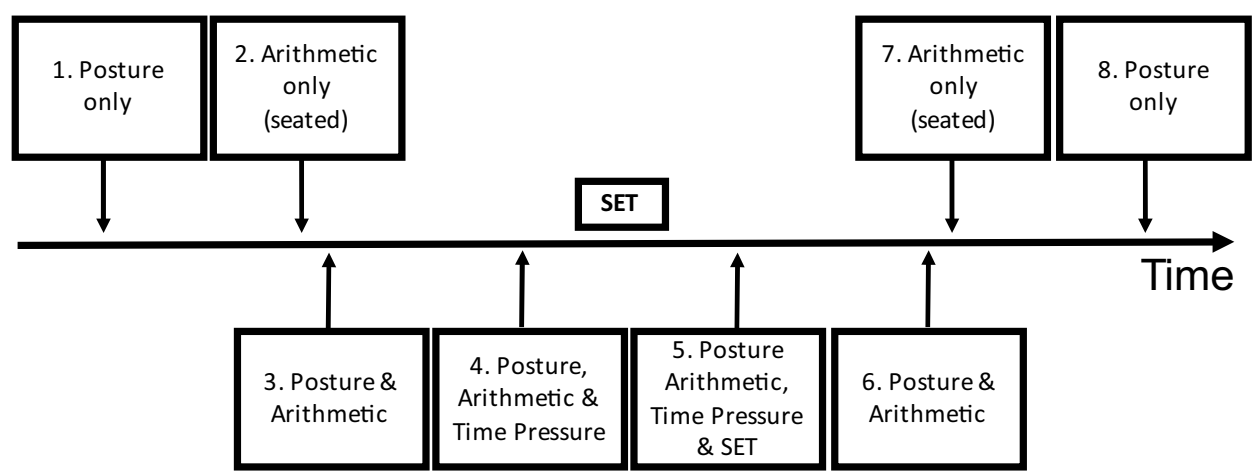

only present in Blocks 4 and 5 (Fig. 1b, c). To induce time pressure, the duration of the green part of the bar was determined as $70 \%$ of the RT in the last block without time pressure (Block 3, see Fig. 1c). After each block, except the first and last posture blocks, participants were asked to provide a $0-10$ rating of how stressed they felt $(0-$ not at all, $10-$ extremely stressed).

\section{Procedure}

Participants responded to an advert for an experiment on 'Effects of maths ability on balance' and were not aware of the SET element of the study. The experimental session was carried out by two experimenters. After obtaining written informed consent and demographic information from the participant, one experimenter left the room. The experiment's timeline is depicted in Fig. 1c. Participants were first asked to perform a 2-min posture trial without the arithmetic task (Block 1). Then, they sat at a table and performed a practice block of 6 trials of the arithmetic task without time pressure followed by a full block of 32 trials of the same task (Block 2) to establish a baseline performance in this task. This was followed by performing the posture task and the arithmetic task concurrently without the progress bar (Block 3 ) and then by performing the posture and arithmetic tasks concurrently with the progress bar (Block 4). The green part of the bar was determined as $70 \%$ of the participant's mean Reaction Time of correct responses in Block 3; however, participants were told that the green part was determined by the average RT of the previous participants.

At the end of Block 4, the Social Evaluative Threat manipulation was implemented. The experimenter inside the room left the room for a few seconds and returned with the other experimenter who pretended to look at the data on a screen that the participant could not see. One of the experimenters then turned to the participant, informed them that they were underperforming in the arithmetic task and asked them to perform better in the next block (Block 5), which comprised the same tasks as the previous block (Block 4) but with a new set of arithmetic problems. Both experimenters closely observed the participant performing the task in this block. Following the SET manipulation, participants performed the three initial blocks again in reverse order to account for practice and possible fatigue effects (Fig. 1c). All participants performed this series of blocks in a fixed order (Blocks 1-8). In the end of the experiment, participants were fully debriefed both in writing and verbally about the purpose of the experiment. 


\section{Data analysis}

In the arithmetic task, we calculated accuracy as the percentage of correct responses and reaction times (RT). In the postural control task, COP data from the Neurocom system in the AP direction were low pass filtered at $4 \mathrm{~Hz}$ using a fifth-order Butterworth dual-pass filter. The first $5 \mathrm{~s}$ of each 2-min block were considered as a stabilisation phase and were discarded. In blocks including simultaneous arithmetic and posture performance, only posture data for the time during which both tasks were performed were analyzed. This was because it was not possible to synchronise the two tasks to end at the same time without changing the number of stimuli in the arithmetic task or the length of the balance trial, given that in participants exhibiting faster RTs, the 32 trials ended earlier. Postural sway was assessed by calculating COP AP and ML amplitude (the maximum-minimum position of the COP), standard deviation (SD), and mean power frequency (MPF). However, we did not expect changes in sway in the ML direction in this task, because sway reference was applied only in the AP direction. Indeed, no differences were observed for ML in neither of the three variables; thus, we only report results in the AP direction. Data in blocks that were repeated before and after the SET manipulation (Blocks 1 and 8, 2, and 7, 3, and 6) were averaged to account for practice or fatigue effects. In addition, to assess the presence of practice or fatigue effects, we contrasted performance in these pairs of blocks using pairedsamples $t$ tests. Our main analyses used repeated measures analyses of variance (ANOVAs) with condition (arithmetic/ posture only; posture and arithmetic; posture, arithmetic and time pressure; and posture, arithmetic, time pressure, and SET) as the within-subjects variables. We were interested in the way that our variables were affected by the addition of each level of task demands, starting with the balance task and adding the arithmetic task, the progress bar and finally SET. Thus, we performed planned comparisons only between successive conditions using pairwise $t$ tests corrected for the three comparisons $(\alpha=0.017)$.

\section{Results}

In this section, we report statistical analyses for self-rated stress, postural control (AP amplitude, SD and MPF), and performance in the arithmetic task (RTs and accuracy).

\section{Self-rated stress}

Results for self-rated stress are depicted in Fig. 2. A repeated measures ANOVA for condition (arithmetic only; posture and arithmetic; posture, arithmetic, and time pressure; and posture, arithmetic time pressure, and SET) showed that

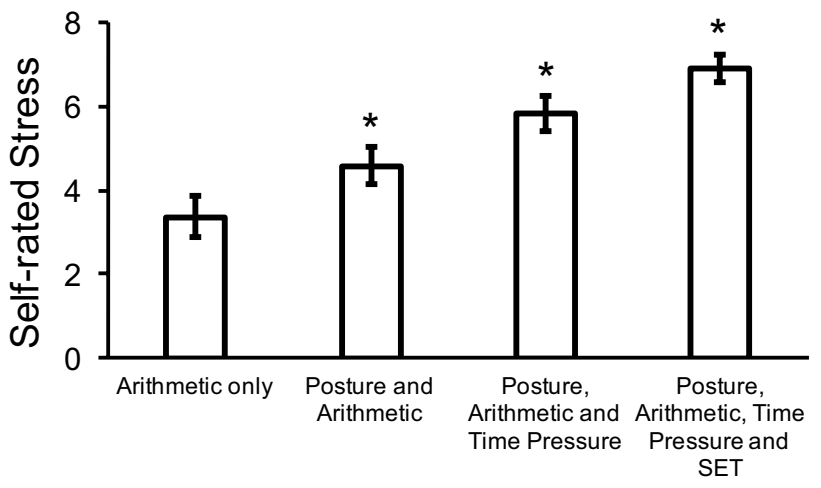

Fig. 2 Self-rated stress values in the four conditions $(0=$ no stress, $10=$ high stress). Error bars represent \pm standard error of the mean (SEM). * $p<.017$ compared to the previous condition

stress increased with condition as shown by a main effect of condition $F(3,33)=47.15, p<0.001, \eta^{2}=0.81$. Planned comparisons contrasting successive conditions showed that all three differences were significant $[t \mathrm{~s}(11)=4.24,5.15$, 3.22 , all $p s<0.008]$. Comparisons of blocks repeated before and after the SET manipulation showed that self-rated stress was not different between the two arithmetic only blocks (Block 2: mean 3.25, SD 1.66; Block 7: mean 3.5, SD 1.83; $t(11)=0.9, p=0.4)$ and the two balance and arithmetic blocks (Block 3: mean 4.5, SD 1.83; Block 6: mean 4.7, SD 1.1.78; $t(11)=0.3, p=0.77)$.

\section{Postural control}

Postural sway AP amplitude, SD, and MPF results are depicted in Fig. 3. The ANOVA for AP amplitude (Fig. 3A) showed a main effect of condition $F(3,33)=5.55, p=0.003$, $\eta^{2}=0.335$. Planned comparisons contrasting successive conditions (Fig. 3a) showed no differences between the posture only and the posture and arithmetic conditions, but showed an increase in amplitude when time pressure was introduced $t(11)=3.41, p=0.006$, followed by a decrease in amplitude from the time pressure to the SET condition $t(11)=3.54$, $p=0.005$. Contrasts between blocks repeated before and after the SET manipulation showed that AP amplitude was not different between the two posture only blocks [Block 1: mean $9.28 \mathrm{~cm}$, SD $4.25 \mathrm{~cm}$; Block 8: mean $7.6 \mathrm{~cm}, \mathrm{SD}$ $4.78 \mathrm{~cm} ; t(11)=1.6, p=0.14]$, but amplitude was greater after the SET manipulation compared with before [Block 3: mean $5.94 \mathrm{~cm}$, SD $3.37 \mathrm{~cm}$; Block 6: mean $7.31 \mathrm{~cm}, \mathrm{SD}$ $4.14 \mathrm{~cm} ; t(11)=2.23, p=0.047]$.

AP SD showed a similar pattern of results (Fig. 3b) and a main effect of condition $F(3,36)=6.42, p=0.002$, $\eta^{2}=0.37$. Pairwise comparisons, however, showed that the only reliable difference was the decrease in SD between 

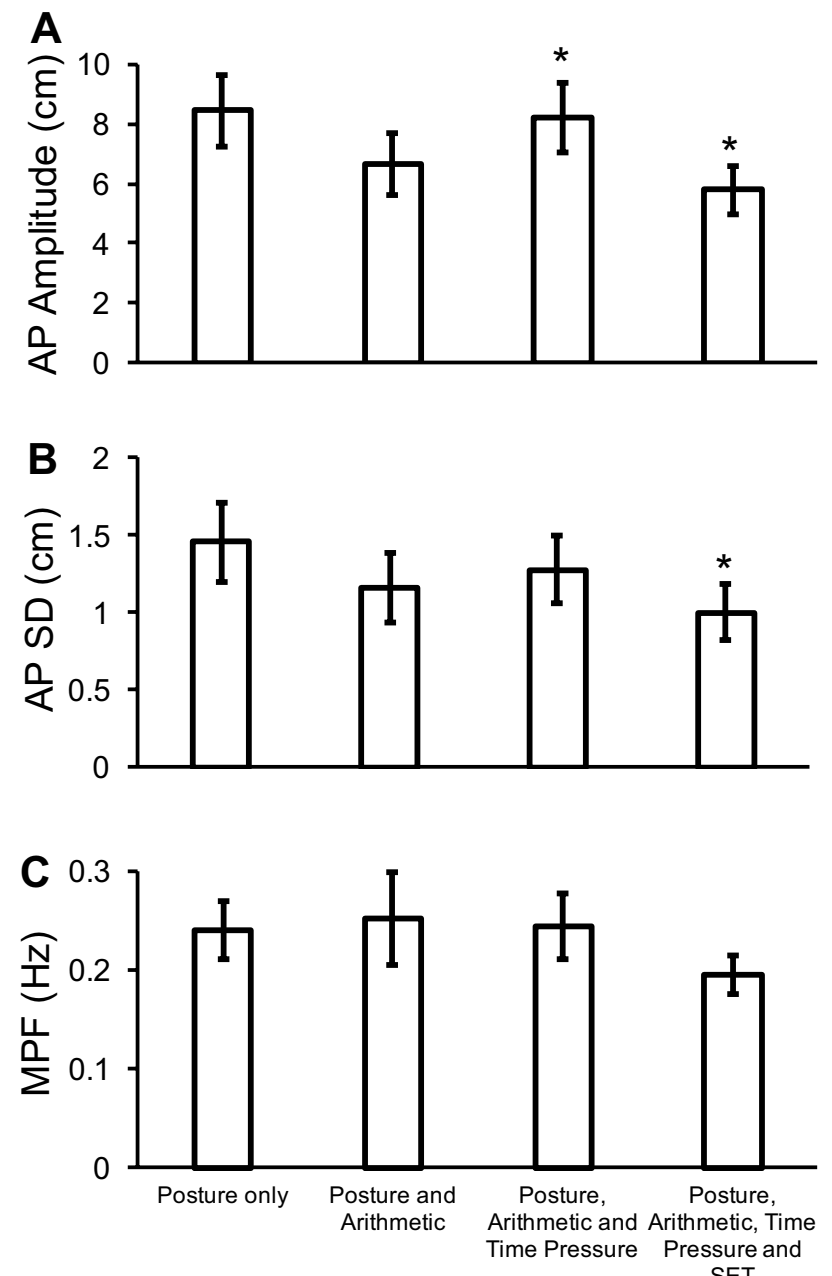

SET

Fig. 3 Postural control results. a AP amplitude, b AP SD, and $\mathbf{c}$ mean power frequency (MPF). Error bars represent \pm standard error of the mean (SEM). $* p<.017$ compared to the previous condition

the last two conditions $t(11)=3.43, p=0.006$. Contrasts between blocks repeated before and after the SET manipulation showed that AP SD was not different between the two posture only blocks [Block 1: mean $1.59 \mathrm{~cm}$, SD $0.88 \mathrm{~cm}$; Block 8: mean $1.32 \mathrm{~cm}$, SD $1.02 \mathrm{~cm} ; t(11)=1.38$, $p=0.2]$ and there were no differences between the two blocks assessing posture and arithmetic [Block 3: mean $1.01 \mathrm{~cm}$, SD $0.78 \mathrm{~cm}$; Block 6: mean $1.22 \mathrm{~cm}$, SD $0.82 \mathrm{~cm}$; $t(11)=1.96, p=0.076]$. Finally, the ANOVA for MPF (Fig. 3c) was not significant. Comparisons between blocks repeated before and after the SET manipulation showed that there was no difference in MPF between the two posture only blocks [Block 1: mean $.26 \mathrm{~Hz}, \mathrm{SD} 0.12 \mathrm{~Hz}$; Block 8: mean .21 Hz, SD $0.1 \mathrm{~Hz} ; t(11)=1.67, p=0.12$ ], but MPF was higher after the SET manipulation compared with before [Block 3: mean $.18 \mathrm{~Hz}, \mathrm{SD} 0.07 \mathrm{~Hz}$; Block 6: mean $.23 \mathrm{~Hz}, \mathrm{SD} 0.1 \mathrm{~Hz} ; t(11)=2.36, p=0.038]$.
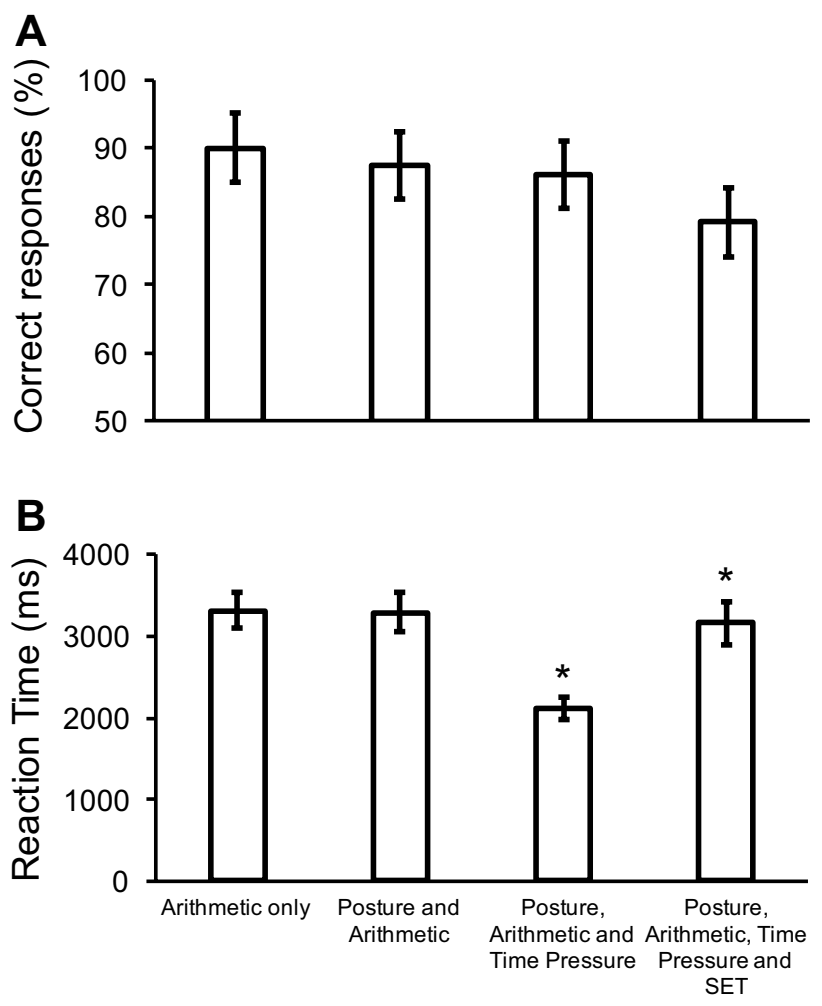

Fig. 4 Arithmetic task performance. a Accuracy and $\mathbf{b}$ reaction time results. Error bars represent \pm SEM. ${ }^{*} p<.017$ compared to the previous condition

\section{Arithmetic}

The two measures of the arithmetic task, accuracy and RT (Fig. 4) were analyzed using separate repeated measures ANOVAs with condition as factor (arithmetic only; posture and arithmetic; posture, arithmetic and time pressure; and posture, arithmetic time pressure, and SET) followed by planned comparisons. Accuracy (Fig. 4a) showed a decrease with condition as shown by a main effect of condition $F(3,33)=5.83, p=0.003, \eta^{2}=0.35$; however, none of the pairwise comparisons between successive blocks were significant $[t \mathrm{~s}(11)=1.66,0.7,1.88, p \mathrm{~s}=0.13,0.5$, 0.09]. Contrasts between blocks repeated before and after the SET manipulation showed that accuracy was not different between the two arithmetic only blocks [Block 2: mean $90.37 \%$, SD $10.78 \%$; Block 7: mean $89.84 \%$, SD $11.24 \%$; $t(11)=0.24, p=0.8$ ] but increased between the two posture and arithmetic blocks [Block 3: mean $81.25 \%$, SD 18.46\%; Block 6: mean 93.75\%, SD 6.53\%; $t(11)=2.55, p=0.027]$.

Reaction time (Fig. 4b) showed similar values between sitting and standing without the progress bar. However, when the bar was introduced (posture, arithmetic, and time pressure condition) reaction times became faster, but became slower again after SET was introduced (bar2 condition). This pattern of results was confirmed by a main effect of 
condition $F(3,33)=25.47, p<.001, \eta^{2}=0.70$, followed by planned comparisons which confirmed the faster RTs when time pressure was first introduced $t(11)=6.08, p<.001$, and then the slower RTs when SET was introduced $t(11)=6.26$, $p<.001$. Comparisons between blocks repeated before and after the SET manipulation showed that RTs were not different in the beginning and in the end of the experiment [Block 2: mean 3153.8, SD 560.11; Block 7: mean 3479.9, SD $1092.04 ; t(11)=1.31, p=0.2]$ but faster RTs were observed before and after the SET manipulation [Block 3: mean 4116.78 ms, SD 1350.38 ms; Block 6: mean 2471.98, $\mathrm{SD} 684.22 \mathrm{~ms} ; t(11)=4.47, p<.001]$.

\section{Discussion}

The aim of this study was to assess whether a reduction in sway is observed when stress increases using a combination of mental arithmetic and SET manipulations. Results showed that when SET was introduced in the context of an arithmetic task, postural sway amplitude and SD decreased, confirming our main hypothesis. To achieve a sufficient increase in stress, we used a gradual introduction of task demand comprising postural, cognitive, and SET elements and we showed that stress increased with task demand in our four conditions. We also assessed whether performance in the arithmetic task was affected by the gradual increase in task demand. As predicted, RT became faster when the progress bar was introduced, suggesting that the time pressure manipulation was successful. However, when SET was introduced RT became slower, suggesting that the high stress induced by the SET impeded performance in the arithmetic task. This increase in RT was accompanied by a decrease in accuracy, although this effect did not reach significance.

This study is the first to demonstrate that SET results in a reduction of postural sway. Our results suggest that the emotional contexts that affect postural sway may not only be postural threat and passive viewing of aversive images but also stress induced using cognitive and SET contexts. We show a reduction in sway with SET, which is in line with studies on postural threat (Carpenter et al. 1999, 2001; Adkin et al. 2000) and aversive or threatening images (Azevedo et al. 2005; Hagenaars et al. 2012; Roelofs et al. 2010), suggesting that the reduction in sway in the present study could be evidence for a common 'freezing' or 'stiffening' mechanism in response to increased threat, anxiety, and stress. However, it is important to acknowledge that to make a strong claim about a stiffening or freezing strategy, it is critical to also show an increase in sway frequency, but our results did not show this increase. Furthermore, we also observed an unexpected increase in sway amplitude when time pressure was introduced. However, both these findings can be interpreted in the context of cognitive resource allocation, and its well-established interactions with postural control (Boisgontier et al. 2013) and stress (Eysenck and Calvo 1992).

There is a large body of evidence (for a review, see Boisgontier et al. 2013), suggesting that postural control relies on cognitive resources. This reliance is primarily used by older adults - who use cognitive resources to prioritize balance using a 'posture first strategy' to avoid falls—but also by young adults (Maylor et al. 2001; Doumas et al. 2008a; Smolders et al. 2010; Doumas and Krampe 2015). In the present study, we used an approach similar to the previous posture-cognitive dual-task studies. However, we introduced increasing cognitive task demands as a way to increase stress (Pruessner et al. 2008; Dedovic et al. 2013). Thus, due to the involvement of both cognitive resource allocation and stress in this study, our results can be interpreted in the context of theories of anxiety and cognition, namely, processing efficiency theory (Eysenck and Calvo 1992). According to this theoretical framework, during relatively simple tasks, performers can compensate for anxiety-related inefficiencies through increasing cognitive effort. However, as task demands increase, such inefficiencies can no longer be compensated for and performance starts to show deficits. We argue that this theoretical approach can be used to interpret our results for at least the first three conditions. In the first two conditions, we observed an increase in stress, but this increase did not affect performance in posture or arithmetic tasks, because the increase in stress-related attentional inefficiencies was compensated for by greater cognitive effort. However, in the third condition, when task demands increased by means of introducing time pressure, stress increased further, along with a reduction in RT and increased postural sway. This result suggests that when time pressure was introduced, additional cognitive resources were directed to the cognitive task to accommodate the increased task demands. Finally, when SET was introduced cognitive and postural task demands were the same but stress increased further, thereby exacerbating anxiety-related processing inefficiencies (Eysenck and Calvo 1992). This increase in stress could not be compensated for through the allocation of cognitive resources, and as a result, RTs became longer and the postural control system triggered a reduction in sway resembling a freezing or stiffening strategy similar to the previous studies. The question remains how to interpret changes in balance control during the most challenging condition. It is possible that reductions in sway could be purely due to arousal-based physiological stiffening adaptations. However, this explanation appears incomplete due to the absence of changes in sway frequency and the further observation that adaptations to sway did not change in line with increased self-reported stress. A more likely explanation could be that reduced sway represents a shift of resources back towards postural control in an attempt to 
restrict unwanted destabilizing movements. This suggestion is supported by increases in RT in the most demanding condition.

Our study had a number of limitations. Stress was primarily measured using self-report. Support for our choice of self-report arises from recent evidence showing that when standing on an elevated surface, self-reported fear is positively correlated with physiological changes such as changes in vestibular reflex gains (Naranjo et al. 2016). However, the previous studies assessing emotional contexts in postural control have used both self-report and physiological measures of emotion and arousal. For example, studies assessing postural threat and fear of falling have primarily used electrodermal activity which measures skin conductance, showing that it is a reliable measure of postural threat (Cleworth et al. 2012; Osler et al. 2013). On the other hand, studies using passive viewing of threatening images used heart rate, based on the idea from the animal literature that freezing is accompanied by bradycardia (Azevedo et al. 2005; Hagenaars et al. 2012; Roelofs et al. 2010). Furthermore, studies assessing stress using social evaluative threat have used cortisol measures (e.g., Dedovic et al. 2013). The addition of one or more of these measures, especially heart rate, would have been useful in strengthening the results of the present study in terms of whether our findings are in line with a freezing response in response to SET. In addition, subjective task difficulty, and the subjective effort allocated to the different aspects of the task was not measured.

A final limitation of our study was that, due to the gradual nature of our stress manipulation, it was not possible to randomise the order of conditions. Specifically, we could not introduce the progress bar before participants performed the arithmetic task, or the SET task before the progress bar. We believe that this incremental increase in task demands was a strength of our paradigm; however, the fixed order of conditions was likely to induce practice effects which could confound our results. This is the reason why we used an experimental design which assessed individual tasks first (posture or arithmetic), then a combination of tasks and then individual tasks again (ABBA design) similar to our previous studies assessing posture-cognitive dual-task performance (Doumas et al. 2008b, 2009, 2012; Smolders et al. 2010; Doumas and Krampe 2015). However, this solution may not be ideal, because averaging may conceal differences between first and last blocks. Thus, to exclude the possibility of practice effects, we contrasted performance from similar blocks ( 1 and 8, 2 and 7, and 3 and 6) in all variables. Indeed, results showed that postural sway amplitude and variability did not show improvement over the course of the experiment, neither in the comparison between the two posture only blocks in the beginning and in the end of the experiment (Block 1 vs 8 ) nor between the two posture and arithmetic blocks (Block 3 vs 6). This lack of sway reduction through practice suggests that the reduction in sway we observed as a result of SET cannot be attributed to practice effects.

Fear, anxiety, and stress are emotions that are highly prevalent in daily life. For example, math anxiety is more prevalent in women than men (Dowker et al. 2016; SuárezPellicioni et al. 2016) and it would be instructive to assess, in a larger sample than in the present study, whether the induced math anxiety using our paradigm and the subsequent changes in postural control are different in women and men. Furthermore, identifying the manner in which emotion and balance interact is important in addressing balance problems and falls in older adults. Older adults at high risk of falling often self-report higher rates of fear of falling as well as movement self-consciousness (Wong et al. 2009), which, in turn, is related to a postural stiffening strategy (Huffman et al. 2009; Zaback et al. 2015). Therefore, using the approach developed for the present study could be used to gradually increase stress in older adults to assess postural responses in a controlled and safe laboratory setting. It is possible that older adults' postural control will be more sensitive to stress manipulations compared with young adults', especially when SET is introduced. Using this paradigm in an age-comparative context would also be very useful in assessing age differences in the relationship between anxiety or fear of falling and associated stiffening. Future research could also assess whether the well-established anxiety-related reduction in sway under stress is adaptive or maladaptive in terms of whether it increases or decreases susceptibility to a fall in young but also in older adults. In general, stiffening is considered a conservative strategy used to prepare the body for a potential disturbance (Young and Williams 2015). It is thought that such adaptations are likely to be beneficial during relatively static balance tasks, but maladaptive in situations, where dynamic responses or preplanned movements are required. Therefore, future research needs to assess whether this stiffening or freezing strategy observed under stress protects a person from a fall after a real-life perturbation, for example, when a bus starts to move or when a person is 'bumped' in a crowded room. Further research in this direction is needed to identify ways in which emotional contexts affect postural control in real-life settings and fall-prone populations.

Acknowledgements The authors wish to thank Conall Curran and Megan Keenan for their assistance in data collection.

\section{Compliance with ethical standards}

Conflict of interest The authors have no conflict of interest to report.

Open Access This article is distributed under the terms of the Creative Commons Attribution 4.0 International License (http://creativecommons.org/licenses/by/4.0/), which permits unrestricted use, 
distribution, and reproduction in any medium, provided you give appropriate credit to the original author(s) and the source, provide a link to the Creative Commons license, and indicate if changes were made.

\section{References}

Adkin AL, Frank JS, Carpenter MG, Peysar GW (2000) Postural control is scaled to level of postural threat. Gait Posture 12:87-93

Azevedo TM, Volchan E, Imbiriba LA et al (2005) A freezing-like posture to pictures of mutilation. Psychophysiology 42:255-260. https://doi.org/10.1111/j.1469-8986.2005.00287.x

Balasubramaniam R, Wing AM (2002) The dynamics of standing balance. Trends Cogn Sci 6:531-536

Black FO, Wall C 3rd, Nashner LM (1983) Effects of visual and support surface orientation references upon postural control in vestibular deficient subjects. Acta Otolaryngol 95:199-201. https:// doi.org/10.3109/00016488309130936

Boisgontier MP, Beets IAM, Duysens J et al (2013) Age-related differences in attentional cost associated with postural dual tasks: increased recruitment of generic cognitive resources in older adults. Neurosci Biobehav Rev 37:1824-1837. https://doi. org/10.1016/j.neubiorev.2013.07.014

Brooks AW (2014) Get excited: reappraising pre-performance anxiety as excitement 143:1144-1158. https://doi.org/10.1037/a0035325

Carpenter MG, Frank JS, Silcher CP (1999) Surface height effects on postural control: a hypothesis for a stiffness strategy for stance. J Vestib Res 9:277-286

Carpenter MG, Frank JS, Silcher CP, Peysar GW (2001) The influence of postural threat on the control of upright stance. Exp Brain Res 138:210-218

Clark S, Riley MA (2007) Multisensory information for postural control: sway-referencing gain shapes center of pressure variability and temporal dynamics. Exp Brain Res 176:299-310. https://doi. org/10.1007/s00221-006-0620-6

Cleworth TW, Horslen BC, Carpenter MG (2012) Influence of real and virtual heights on standing balance. Gait Posture 36:172-176. https://doi.org/10.1016/j.gaitpost.2012.02.010

Davis JR, Horslen BC, Nishikawa K et al (2011) Human proprioceptive adaptations during states of height-induced fear and anxiety. J Neurophysiol 106:3082-3090. https://doi.org/10.1152/ jn. 01030.2010

de Groot MH, van Campen JPCM, Moek MA et al (2013) The effects of fall-risk-increasing drugs on postural control: a literature review. Drugs Aging 30:901-920. https://doi.org/10.1007/ s40266-013-0113-9

Dedovic K, Renwick R, Mahani NK, Engert V (2005) The Montreal imaging stress task: using functional imaging to investigate the effects of perceiving and processing psychosocial stress in the human brain. Psychiatry Neurosci 30:319-325

Dedovic K, Duchesne A, Engert V et al (2013) Psychological, endocrine and neural responses to social evaluation in subclinical depression. Soc Cogn Affect Neurosci 9:1632-1644. https://doi. org/10.1093/scan/nst151

Dickerson SS, Kemeny ME (2004) Acute stressors and cortisol responses: a theoretical integration and synthesis of laboratory research. Psychol Bull 130:355-391. https://doi. org/10.1037/0033-2909.130.3.355

Doumas M, Krampe RT (2010) Adaptation and reintegration of proprioceptive information in young and older adults' postural control. J Neurophysiol. https://doi.org/10.1152/jn.00345.2010

Doumas M, Krampe RT (2015) Ecological relevance determines task priority in older adults' multitasking. J Gerontol Ser B Psychol Sci Soc Sci. https://doi.org/10.1093/geronb/gbt105
Doumas M, Smolders C, Krampe RT (2008a) Task prioritization in aging: effects of sensory information on concurrent posture and memory performance. Exp Brain Res 187:275-281

Doumas M, Wing AM, Wood K (2008b) Interval timing and trajectory in unequal amplitude movements. Exp Brain Res 189:49-60

Doumas M, Rapp MA, Krampe RT (2009) Working memory and postural control: adult age differences in potential for improvement, task priority, and dual tasking. J Gerontol Ser B Psychol Sci Soc Sci 64:193-201

Doumas M, Smolders C, Brunfaut E et al (2012) Dual task performance of working memory and postural control in major depressive disorder. Neuropsychology. https://doi.org/10.1037/a0026181

Dowker A, Sarkar A, Looi CY (2016) Mathematics anxiety: what have we learned in 60 years? Front Psychol. https://doi.org/10.3389/ fpsyg.2016.00508

Eysenck MW, Calvo MG (1992) Anxiety and performance: the processing efficiency theory. Cogn Emot 6:409-434. https://doi. org/10.1080/02699939208409696

Eysenck MW, Santos R, Calvo MG (2007) Anxiety and cognitive performance. Attent Control Theory 7:336-353. https://doi. org/10.1037/1528-3542.7.2.336

Facchinetti LD, Imbiriba LA, Azevedo TM et al (2006) Postural modulation induced by pictures depicting prosocial or dangerous contexts. Neurosci Lett 410:52-56. https://doi.org/10.1016/j. neulet.2006.09.063

Frisch JU, Häusser JA, Mojzisch A (2015) The trier social stress test as a paradigm to study how people respond to threat in social interactions. 6:1-15. https://doi.org/10.3389/fpsyg.2015.00014

Hagenaars MA, Stins JF, Roelofs K (2012) Aversive life events enhance human freezing responses. J Exp Psychol Gen 141:98-105. https://doi.org/10.1037/a0024211

Hagenaars MA, Oitzl M, Roelofs K (2014) Updating freeze: aligning animal and human research. Neurosci Biobehav Rev 47:165-176. https://doi.org/10.1016/j.neubiorev.2014.07.021

Hillman CH, Rosengren KS, Smith DP (2004) Emotion and motivated behavior: postural adjustments to affective picture viewing. Biol Psychol 66:51-62. https://doi.org/10.1016/j. biopsycho.2003.07.005

Horslen BC, Carpenter MG (2011) Arousal, valence and their relative effects on postural control. Exp Brain Res 215:27-34. https://doi. org/10.1007/s00221-011-2867-9

Horslen BC, Murnaghan CD, Inglis JT et al (2013) Effects of postural threat on spinal stretch reflexes: evidence for increased muscle spindle sensitivity? J Neurophysiol 110:899-906. https://doi. org/10.1152/jn.00065.2013

Horslen BC, Dakin CJ, Inglis JT et al (2014) Modulation of human vestibular reflexes with increased postural threat. J Physiol 592:36713685. https://doi.org/10.1113/jphysiol.2014.270744

Huffman JL, Horslen BC, Carpenter MG, Adkin AL (2009) Does increased postural threat lead to more conscious control of posture? Gait Posture 30:528-532. https://doi.org/10.1016/j. gaitpost.2009.08.001

Kirschbaum C, Pirke K-M, Hellhammer DH (1993) The "Trier Social Stress Test"-a tool for investigating psychobiological stress responses in a laboratory setting. Neuropsychobiology 28:76-81

Lang PJ, Davis M, Öhman A (2000) Fear and anxiety: animal models and human cognitive psychophysiology. J Affect Disord 61:137159. https://doi.org/10.1016/S0165-0327(00)00343-8

LeDoux JE, Iwata J, Cicchetti P, Reis DJ (1988) Different projections of the central amygdaloid nucleus mediate autonomic and behavioral correlates of conditioned fear. J Neurosci 8:2517-2529

Lim SB, Cleworth TW, Horslen BC et al (2017) Postural threat influences vestibular-evoked muscular responses. 604-611. https://doi. org/10.1152/jn.00712.2016

Maki BE, McIlroy WE (1996) Influence of arousal and attention on the control of postural sway. J Vestib Res 6:53-59 
Maylor EA, Allison S, Wing AM (2001) Effects of spatial and nonspatial cognitive activity on postural stability. Br J Psychol 92:319338. https://doi.org/10.1348/000712601162211

McCollum G, Shupert CL, Nashner LM (1996) Organizing sensory information for postural control in altered sensory environments. J Theor Biol 180:257-270. https://doi.org/10.1006/jtbi.1996.0101

Naranjo EN, Cleworth TW, Allum JHJ et al (2016) Vestibulo-spinal and vestibulo-ocular reflexes are modulated when standing with increased postural threat: 833-842. https://doi.org/10.1152/ jn.00626.2015

Osler CJ, Tersteeg MCA, Reynolds RF, Loram ID (2013) Postural threat differentially affects the feedforward and feedback components of the vestibular-evoked balance response. Eur J Neurosci 38:3239-3247. https://doi.org/10.1111/ejn.12336

Peterka RJ, Black FO (1990) Age-related changes in human posture control: sensory organization tests. J Vestib Res 1:73-85

Peterka RJ, Loughlin PJ (2004) Dynamic regulation of sensorimotor integration in human postural control. J Neurophysiol 91:410-423

Pruessner JC, Dedovic K, Khalili-Mahani N et al (2008) Deactivation of the limbic system during acute psychosocial stress: evidence from positron emission tomography and functional magnetic resonance imaging studies. Biol Psychiatry 63:234-240. https://doi. org/10.1016/j.biopsych.2007.04.041

Roelofs K, Hagenaars MA, Stins J (2010) Facing freeze: social threat induces bodily freeze in humans. Psychol Sci 21:1575-1581. https://doi.org/10.1177/0956797610384746
Smolders C, Doumas M, Krampe RT (2010) Posture and cognition interfere in later adulthood even without concurrent response production. Hum Mov Sci. https://doi.org/10.1016/j. humov.2009.07.009

Stins JF, Beek PJ (2007) Effects of affective picture viewing on postural control. BMC Neurosci 8:83. https://doi. org/10.1186/1471-2202-8-83

Suárez-Pellicioni M, Núñez-Peña MI, Colomé À (2016) Math anxiety: a review of its cognitive consequences, psychophysiological correlates, and brain bases. Cogn Affect Behav Neurosci 16:3-22. https://doi.org/10.3758/s13415-015-0370-7

Van der Kooij H, Campbell AD, Carpenter MG (2011) Sampling duration effects on centre of pressure descriptive measures. Gait Posture 34:19-24. https://doi.org/10.1016/j.gaitpost.2011.02.025

Wong W-L, Masters RSW, Maxwell JP, Abernethy B (2009) The role of reinvestment in walking and falling in communitydwelling older adults. J Am Geriatr Soc 57:920-922. https://doi. org/10.1111/j.1532-5415.2009.02228.x

Young WR, Williams AM (2015) How fear of falling can increase fall-risk in older adults: applying psychological theory to practical observations. Gait Posture 41:7-12. https://doi.org/10.1016/j. gaitpost.2014.09.006

Zaback M, Cleworth TW, Carpenter MG, Adkin AL (2015) Personality traits and individual differences predict threat-induced changes in postural control. Hum Mov Sci 40C:393-409. https://doi. org/10.1016/j.humov.2015.01.015 